\title{
THE INPUT CATALOGUE TESTED BY THE FIRST HIPPARCOS OBSERVATIONS II. THE PHOTOMETRY AND VARIABLE STARS
}

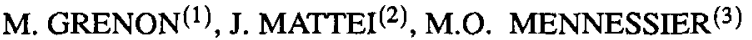 \\ (1) Observatoire de Genève, CH-1290 Sauvemy, Switzerland \\ (2) AAVSO, 25 Birch Street, Cambridge MA 02138, USA \\ (3) Université du Languedoc, F-34060 Montpellier, France
}

ABSTRACT. The methods developed to predict instrumental magnitudes, to monitor the ageing of the optics, to calibrate photometrically the payload and finally to build up a high accuracy photometric reference system are tested against space observations. The quality of ephemerides for long period variables are also discussed.

\section{Introduction}

For the star selection purpose and the observing time allocation, approximate instrumental magnitudes had to be produced for the whole Hipparcos data base of 212000 stars. During the development of the satellite it became patent that more science than initially forseen could be made with the photoelectrons collected by the main mission and the star mapper detectors. Star mappers were then equipped with broad band filters and all detection chains were carefully calibrated photometrically before launch. Rapid changes of the optical transmission were expected due to irradiation by solar and cosmic particles. A detailed calibration process in-orbit had to be defined. It is based on the routine observation of a large number of photoelectric standards with predetermined accurate instrumental magnitudes. $H p, B_{T}, V_{T}$. Ephemerides in terms of instrumental $H p$ magnitude have to be produced for 240 long-period variables. The goodness of an a priori determination of filters, instrumental magnitudes, ageing effects on the optics and quality of the ephemerides are discussed hereafter.

\section{The Hp, $\mathbf{B}_{T}, \mathrm{~V}_{T}$ bands}

The $B_{T}, V_{T}$ bands were determined both from a laboratory photometric calibration of the detection chains and by combining the transmission and reflection of all optical elements with the quantum efficiencies of the detectors. A mean Tycho system was defined with properties intermediate between those of the preceding and following fields of view. The relations between $B_{T}, V_{T}$ and the magnitudes and colours of the current photoelectric systems were estimated through synthetic photometry. The observed intensities are $7.4 \%$ larger than expected. The shape of the bands are as determined, since the chromatic term necessary for the reduction of instantaneous magnitudes of the standard system, is in the mean as small as $0.005 \cdot(B-V)$.

The adopted description of the main mission band was that measured in laboratory. A nonnegligible difference between the predicted and observed $H p$ was noticed. At the epoch of launch, the corrective term for the reduction of a standard was $0.066 \cdot(\mathrm{B}-\mathrm{V})$. The difference in the quantum efficiency of the $H p$ arises from the choice of the redundant chains instead of the nominal for which the standard was defined and by an extended sensitivity in the near IR up to $900 \mathrm{~nm}$. As 
a consequence of optic ageing, this chromatic term was as high as 0.100 in July 1991 , so that the precision on the reduced $H p$ magnitude became limited by the uncertainties on the star colours. A redefinition of the $H p$ system has to be forseen, as well as of colour index, the Cousins (V-I) will be used instead of (B-V) in order to maintain the systematic errors, colour dependent, below 0.001 mag for the reddest stars.

\section{The ageing of the optics}

The irradiation tests performed on the components of the optics predicted transmission losses of about $6 \%$ per year due to cosmic rays, plus possibly up to $3 \%$ more in case of major solar flares, with a satellite on a geostationary orbit. In the case of the revised mission and the regular crossing of van Allen belts, the solar particles effect became more important. Transmission loss, for (B$\mathrm{V})=0.5$ stars, turns out to be of the order of $14 \%$ per year; it is smoother than expected and will lead to a lowering of the limiting magnitude by $0.48 \mathrm{mag}$ and 0.80 mag after 3 and 5 years mission respectively.

Chromatic changes behave as estimated with blue stars more attenuated than yellow or red ones. In July 1991, the raw magnitude increase was $0.30 \mathrm{mag}$ for $(\mathrm{B}-\mathrm{V})=0.0 \mathrm{stars}$ and only $0.20 \mathrm{mag}$ for $(\mathrm{B}-\mathrm{V})=1.50$ stars. The changes on $B_{T}, V_{T}$ channels are very small as forseen since the materials are less radiation sensitive.

The optical transmission behaves quantitatively and qualitatively as computed and no modification of the observing programme was necessary.

\section{The achievable accuracy on $H p$}

In spite of the small collecting surface, i.e. half a $29 \mathrm{~cm}$ telescope, the absence of scintillation and atmospherical absorption allows high accuracy photometry with the main mission detector. The single transit precision ranges from $0.006 \mathrm{mag}$ to 0.024 for $\mathrm{Hp}=6$ and 9 mag respectively. With about 200 measurements per star, the achievable acccuracy on the mean $H p$ is extremely high for constant stars:

\begin{tabular}{rcccc}
$H p:$ & 6 & 8 & 10 & 12 \\
\hline se $H p:$ & .0006 & .0015 & .004 & .017
\end{tabular}

Ageing effects induce magnitude changes of about +0.013 mag between two consecutive groups of field of view crossings. To maintain the magnitude scale drift at the submilimagnitude level requires a close monitoring of instrumental changes as well as a detailed description of the bidimensional sensitivity of the detection chains. These conditions are now fulfilled by a complex calibration using calibrating stars.

\section{The calibrating stars}

Stars suitable for calibration purpose were selected among programme stars having instrumental magnitudes accurately defined from multicolour photometry. A number of 150400 sets of multicolour data were treated to produce $H p, B_{T}, V_{T}$ for 46000 programme stars. Those with a standard error on $H p$ less than 0.020 mag and with confirmed stability were retained as candidates for the standard. Multiple stars and possible microvariables were rejected and a final sample of 13580 standards was finally retained. For $65 \%$ of standards, the standard error on $m v$ was less than 0.010 mag. 
The quality of the main mission standard was tested on-orbit and its external accuracy estimated. For bright stars, the uncertainties due to ground-based measurement errors and inadequacies of transformation equations dominate over the photon noise and reduction errors of the Hipparcos photometry. The accuracy of the standard was estimated by Mignard (1991) to be in the range 0.012 to $0.018 \mathrm{mag}$ depending on the declination.

With about 600 standards per great circle the zero point of the $H p$ magnitude scale is then determined with an accuracy better than $0.001 \mathrm{mag}$ all along the mission.

After the reduction of about one year's data, the standard will be updated and extended. The stars demonstrated as constant will have their predicted $H p$ magnitudes replaced by the mean $H p$ observed from space in order to reduce the noise on calibrating stars by a factor 10 . Additional stars also constant at a few millimagnitude level will be included in the standard if their colours are well defined. This process will ensure a uniform density of standards and the removal of local systematic errors.

A distinct set of 25000 standards labelled in terms of $B_{T}, V_{T}$ magnitude was also prepared for the calibration of Tycho.

\section{The variable stars detection}

Since the detection efficiency changes are precisely monitored and the optical properties of the instrument exactly modelled, variable stars with extremely low amplitude may be detected. The threshold Amin is indeed magnitude dependent as shown below for stars with sinusoidal light variations:

\begin{tabular}{ccccc}
$H p:$ & 6 & 8 & 10 & 12 \\
\hline Amin : & .007 & .017 & .048 & .20
\end{tabular}

Periods in the range of a few minutes to several years are also detectable. With such low detection thresholds, most programme stars will appear as microvariables. In addition to pulsation effects, micro flares or effects related to the stellar rotation as spots distribution or marginal $A p$ phenomenon are within the scope of the Hipparcos photometry. Secular luminoisty variations as small as 0.001 $\mathrm{mag} /$ year should be detectable.

\section{A new photometric reference system}

Once the calibration process is applied to all Hipparcos programme stars, a dense net of high accuracy mean $H p$ magnitude will be defined over the whole sky with a uniform density. The adopted scanning law guarantees that at the end of the mission, all systematic residual errors, function of the right ascension and declination, of the standard will be eliminated. After removal of variable stars and binaries , every point of the new reference system should be characterized by a mean magnitude, with a precision from less than 0.001 to a few millimagnitudes depending on the level of micro-variability and the magnitude. This system will supercede the various photometric standards now available.

\section{The long-period variables}

The long-period variables represent only $0.2 \%$ of the Hipparcos programme. Nevertheless, they imply a considerable observational effort for the ground-based observers coordinated by AAVSO. A number of 70000 observations per year are obtained for the 240 LPVs. Visual observations are 
transformed into instrumental magnitudes at Montpellier where ephemerides are produced every two months. The extrapolation of light-curves is based on Fourier transform and learning rules to describe the departures from period curves. The specified acccuracy for the magnitude prediction is $0.5 \mathrm{mag}$. At the beginning of the mission this specification was verified for $75 \%$ of the variables. Longer time series and tuning of prediction methods have increased the rate of successful prediction to $85 \%$.

The comparison of predicted magnitudes with the raw $H p$ magnitude from the satellite reveals a colour-dependent discrepancy of 0.1 to $0.6 \mathrm{mag}$. A detailed analysis of the data has shown that the visual magnitudes produced by the amateur astronomer do not deviate by more than $0.1 \mathrm{mag}$ with respect to photoelectric magnitude for LPVs. The difference was due, half to the enhanced IR sensitivity of the detector and half to the magnitude extraction method applied to samples in the quick look procedure. In the field of LPVs too, the methods developed during the mission preparation are validated.

\section{References}

Grenon, M., 1989, ESA SP-1111, pp. 129-140 and pp. 141-147

Mennessier, M.O., Figueras, F., 1989, ESA SP-1111, pp. 163-176

Mignard, F., 1991, private communication 